\title{
Inappropriate sinus tachycardia in long-COVID and other updates on recent autonomic research
}

\author{
Mitchell G. Miglis ${ }^{1} \cdot$ Nicholas Larsen $^{1} \cdot$ Srikanth Muppidi ${ }^{1,2}$
}

Received: 20 January 2022 / Accepted: 20 January 2022 / Published online: 7 February 2022

(c) The Author(s), under exclusive licence to Springer-Verlag GmbH Germany 2022

\section{Inappropriate sinus tachycardia in long-COVID}

As the COVID-19 pandemic continues to affect hundreds of millions of people worldwide, autonomic complications are being recognized with increasing frequency in both the acute and chronic form of the illness, the latter now designated as post-acute sequelae of SARS-CoV-2 (PASC), colloquially termed long-COVID. Tachycardia is a common symptom of PASC, and some patients may be diagnosed with postural tachycardia syndrome (POTS) or inappropriate sinus tachycardia (IST). While post-COVID POTS has garnered more attention from the scientific and popular press, IST may be equally or more common and has been reported in survivors of the prior SARS coronavirus pandemic [1]. The etiology of IST is unknown; however, the proposed mechanisms overlap with those of POTS, including increased sympathetic and/or reduced cardiovagal activity.

In their recent publication [2], "Inappropriate sinus tachycardia in post-COVID-19 syndrome" Aranyo and colleagues identified 200 consecutive patients with PASC. IST was defined according to consensus criteria as symptomatic sinus rhythm rate $\geq 100 \mathrm{bpm}$ at rest with a mean 24 -h heart rate above 90 beats/min in the absence of any acute physiological demand or conditions known to produce sinus tachycardia. All patients underwent a 10-min active stand test to help rule out disorders of orthostatic intolerance, such as POTS and orthostatic hypotension. In addition, patients underwent an echocardiogram, 24-h Holter monitoring, a 6-min walk test (6MWT), and measurements of blood markers of inflammation and myocardial damage, including ferritin and interleukin-6. Heart rate variability (HRV) analysis was

Srikanth Muppidi

muppidis@stanford.edu

1 Stanford Medical Center, Palo Alto, CA, USA

2 Stanford Neurosciences Health Center, 213 Quarry Road, 2nd Floor, Palo Alto, CA 94304, USA performed from the 24-h Holter data, focusing on standard time-domain parameters such as the RR interval, standard deviation of the inter-beat interval (SDNN), and the percentage of adjacent NN intervals that differed from each other by more than $50 \mathrm{~ms}$ (PNN50), as well as standard frequencydomain parameters including very low-frequency (VLF; 0.003-0.04 Hz), low-frequency (LF; 0.04-0.15 Hz), and high-frequency (HF; $0.15-0.40 \mathrm{~Hz}$ ) bands. The HF band and PNN50 are regarded as indicators of the parasympathetic influence on heart rate, whereas the LF and VLF bands have more complex physiology that integrates both the sympathetic and parasympathetic components. Comparison groups included age- and gender-matched PCR-confirmed COVID patients without IST (fully recovered group, $N=19$ ) and age- and gender-matched controls who had no history of SARS-COV-2 disease, as confirmed by negative serology (uninfected group, $N=17$ ).

The authors identified 40 patients with IST from the cohort of 200 patients with PASC yielding an estimated prevalence of $20 \%$. Eighty-five percent of the subjects were women and $83 \%$ had a history of mild COVID defined as the presence of symptoms without evidence of viral pneumonia or hypoxia. Most patients reported cardiopulmonary symptoms at the onset of their infection, including palpitations (90\%), dyspnea (83\%), and chest pain $(78 \%) ; 53 \%$ reported dizziness and $48 \%$ anosmia. Compared to fully recovered patients, patients with IST were more likely to report palpitations ( $90 \%$ vs. $5 \% ; p<0.001)$, dyspnea ( $82 \%$ vs. $16 \%$; $p<0.001)$, chest pain (78\% vs. $21 \% ; p<0.001)$, headache (73\% vs. 37\%; $p=0.007)$, dizziness $(53 \%$ vs. $5 \% ; p=0.002)$, diarrhea ( $53 \%$ vs. $16 \% ; p=0.003)$, and dermatological alterations $(35 \%$ vs. $5 \% ; p=0.009)$ during the acute phase of SARS-CoV-2 infection. Patients with IST were also more likely to report environmental allergies compared to the fully recovered and uninfected groups (25\% vs. $0 \% ; p=0.01)$, hinting at a potential mast cell component.

The 6MWT showed that patients with PASC with IST had a significantly reduced exercise capacity, with a $60 \%$ 
reduction in their estimated normal distance. Frequency analysis of heart rate variability revealed that patients with IST had a reduction in VLF, LF, and HF domains compared to controls, with more severe impairment in the parasympathetic-influenced domains. There was no difference in blood inflammatory markers between groups.

Limitations of this work include the small sample sizes of the control groups, as well as the lack of systematic pulmonary evaluations, measurement of markers of physical deconditioning, and systematic autonomic testing. Nonetheless, this study provides the first glimpse of how common IST may be in PASC and adds to the growing body of literature on autonomic dysfunction as a potential mechanism in PASC-related disability. With the Omicron variant currently surging across the globe, PASC-related autonomic complications will, unfortunately, be with us for some time, and clinicians should be familiar with their manifestations and potential treatment options.

\section{Idiopathic small fiber neuropathy: how much autoimmunity?}

Small fiber neuropathy (SFN) is a heterogeneous disease that may present with pain, paresthesias, and sometimes autonomic dysfunction. Although there are known conditions that cause SFN such as diabetes and vitamin B12 deficiency, in up to $50 \%$ of cases no underlying cause can be found [3]. In such idiopathic cases, response to IVIG has been documented in a subset of patients suggesting underlying autoimmunity [4]; however, a recent randomized placebocontrolled trial demonstrated no improvement with IVIG compared to placebo in patients with painful idiopathic SFN [5].

Adding to this body of literature, Chan and colleagues [6] used a novel antibody technology to identify autoantibodies in patients with idiopathic SFN. In this study, two cohorts of patients were recruited. The main cohort was recruited from the Neurology Clinic at the National University Hospital in Singapore and included 59 patients (age $\geq 21$ years) who had SFN based on NEURODIAB criteria. The validation cohort included 36 patients with SFN who were recruited using the same criteria from the National University Hospital in Singapore and Lucerne Cantonal Hospital in Lucerne, Switzerland. These two cohorts of patients were compared to 20 healthy subjects matched by age and gender. Patients with SFN underwent a wide array of serologic testing, nerve conduction studies (NCS), and skin biopsies. Needle electromyography (EMG), quantitative sensory testing (QST), quantitative sudomotor axonal reflex testing (QSART), and tilt table testing were also performed if clinically indicated. Patients with SFN were grouped into "possible SFN" (symptoms and/or signs of SFN), "probable SFN" (clinical signs + normal sural NCS), and "definite SFN" (clinical signs + normal sural NCS + abnormal QST at the foot and/or decreased intraepidermal nerve fibers at the ankle). The most likely underlying etiology of SFN was further reviewed by three neurologists and grouped into idiopathic SNF (iSFN) or SFN by secondary causes (rheumatologic disease, diabetes, nutritional deficiencies, etc.).

Peripheral blood was collected from the patients with SFN in both cohorts, 20 healthy controls and 5 pooled normal sera, and screened for $>1600$ proteins on the Sengenics KREX platform. The samples were analyzed via Sengenics Immunome Protein Array which is a novel high-throughput protein microarray platform that captures autoantibodies expressed in the native conformational state.

In the main cohort, one patient was excluded due to abnormal NCS. Among the remaining 58 patients, there was a slight male predominance (53.4\%), and the mean age was $50 \pm 13.5$ yrs. Forty-three patients $(74.1 \%)$ were diagnosed with definite SFN via positive skin biopsy or abnormal QST in 2 or more limbs. Markers of autoimmunity (ANA greater 1:80, high ESR for age, and presence of anti-ENA or ANCA) were found in $29.3 \%$ of patients with SFN. Idiopathic SFN was diagnosed in 34 patients $(58.6 \%)$ and secondary SFN was diagnosed in 24 patients (41.4\%). Comparisons between the main cohort and validation cohort showed no differences in the prevalence of iSFN, and diagnostic certainty of SFN; however, there were more females in the validation cohort (78.8\%).

Proteomic testing found that in the main cohort, nine autoantibodies (MX1, DBNL, LIN28A, KRT8, METTL3, NCOA5, PTPN1, ZNF276, and MIF) showed reproducibly significant differences compared to controls. Three proteins showed consistently high fold changes in both the main and validation cohorts. These proteins were MX1, DBNL, and KRT8. Subgroup analysis comparing iSFN and SFN by secondary causes showed that MX1 fold change was higher in the iSFN group $(\mathrm{FC}=1.61$ vs $0.106, p=0.009)$.

Most protein purification methods denature the native conformation of the protein which affects the interaction between antigen and antibody which in turn reduced the sensitivity and specificity of antibody detection [7]. This was the first study to utilize a high-throughput protein microarray platform that captures autoantibodies expressed in the native conformational state in patients with SFN. Using this novel technique, the authors were able to identify nine reproducible autoantibodies that are either known to be associated with inflammatory neuropathy and neuropathic pain or are involved in cellular processes of transcription, DNA, and RNA functions and repair [8]. The subgroup analysis identified MX1 as a potential marker to differentiate iSFN from secondary forms of SFN. In summary, this study was able to identify novel antibodies in SFN, providing evidence that 
an immune-mediated process may be involved in a subset of patients with iSFN.

Limitations of this study include the relatively small sample sizes and the retrospective analysis. The study was also limited by missing data which led to the exclusion of patients in the subgroup analysis. Therefore, the proportion of patients with iSFN may not be fully representative of the general population. Future studies should focus on a detailed analysis of the autoantibodies found in this study and validation of their clinical significance.

\section{Autonomic pathways and autoimmunity}

Vagal afferent and efferent pathways play a key role in our ability to perceive immunological changes in the body through the inflammatory reflex [9]. However, how these inflammatory signals are stored in the brain, and the effects of reactivation of such signals have not been fully elucidated. In their recently published work, titled, "Insular cortex neurons encode and retrieve specific immune responses", Koren et al. [10] provide further evidence of how the brain encodes peripheral inflammation. We encourage readers to read the excellent article and associated editorial [11] for details on mice models and various techniques used in the study.

In brief, researchers used two different models of peripheral inflammation in mice (dextran sulfate sodium (DSS)induced model of colitis or a Zymosan-induced model of peritonitis). In this experiment, the study team looked for changes in neuronal activity in the insular cortex-a region of the central autonomic network known to play an important role in interoception and integration of various visceral signals. After inducing colitis or peritonitis in mice, researchers found two distinct types of neuronal encoding and activation in the posterior insular cortex based on the type of peripheral insult (colitis vs. peritonitis). Stunningly, once mice recovered from colitis or peritonitis, the researchers were able to reproduce peripheral inflammation by activating these specific neuronal ensembles in the insular cortex without any peripheral trigger. These findings suggest that immunological "memory engrams", are stored in the insular cortex for different peripheral immune insults and can restore the initial disease state when reactivated.

To understand anatomical pathways that allow for signal transmissions between the insular cortex and peripheral organs, the researchers used polysynaptic viral-labeling techniques with pseudorabies virus and AAV1. These methods showed that retrograde and anterograde signal pathways converge at the dorsal motor nucleus of the vagus and the rostral ventrolateral medulla, suggesting that autonomic pathways are critical for signal transmission of peripheral immunerelated injury. In a final experiment, inhibition of the insular cortex reduced the degree of clinical and immune parameters from experimentally induced colitis.

This elegant work provides clear evidence that the insular cortex provides critical storage of peripheral immune insults and regulates immune responses, and that autonomic pathways in the brainstem are the primary hub for signal transmission. This work also raises several questions including the effect of chronic inflammation on the insular cortex. In addition, given the role of the insula as a hub in emotion regulation and memory, it raises the question of whether other physiological stressors influence these pathways, as many patients report worsening of immune-mediated diseases with stress. Since vagal nuclei and pathways are critical for signal transmission, it would also be interesting to evaluate if interventions with medications or vagal stimulation lead to alteration in the insular cortical influence on peripheral immune responses. Ultimately, we hope that additional research in this field will translate to interventions for patients, and autonomic physicians will likely have a key role to play.

Funding None.

\section{Declarations}

Conflict of interest None.

\section{References}

1. Lau ST, Yu WC, Mok NS, Tsui PT, Tong WL, Cheng SWC (2005) Tachycardia amongst subjects recovering from severe acute respiratory syndrome (SARS). Int J Cardiol 100(1):167-169. https:// doi.org/10.1016/j.ijcard.2004.06.022

2. Aranyó J, Bazan V, Lladós G, et al. Inappropriate sinus tachycardia in post-COVID-19 syndrome. Sci Rep. 2022;12(1):1-9. https://doi.org/10.1038/s41598-021-03831-6

3. Basantsova NY, Starshinova AA, Dori A, Zinchenko YS, Yablonskiy PK, Shoenfeld Y (2019) Small-fiber neuropathy definition, diagnosis, and treatment. Neurol Sci 40(7):1343-1350. https://doi. org/10.1007/s10072-019-03871-x

4. Liu X, Treister R, Lang M, Oaklander AL (2018) IVIg for apparently autoimmune small-fiber polyneuropathy: first analysis of efficacy and safety. Ther Adv Neurol Disord. https://doi.org/10. $1177 / 1756285617744484$

5. Geerts M, de Greef BTA, Sopacua M et al (2021) Intravenous immunoglobulin therapy in patients with painful idiopathic small fiber neuropathy. Neurology 96(20):e2534-e2545. https://doi.org/ 10.1212/WNL.0000000000011919

6. Chan ACY, Wong HY, Chong YF et al (2022) Novel autoantibodies in idiopathic small fiber neuropathy. Ann Neurol 91(1):66-77. https://doi.org/10.1002/ana.26268

7. Mak A, Kow NY, Ismail NH et al (2020) Detection of putative autoantibodies in systemic lupus erythematous using a novel native-conformation protein microarray platform. Lupus 29(14):1948-1954. https://doi.org/10.1177/0961203320959696

8. Bateman A, Martin MJ, Orchard S et al (2021) UniProt: the universal protein knowledgebase in 2021. Nucleic Acids Res 49(D1):D480-D489. https://doi.org/10.1093/nar/gkaa1100 
9. Tracey KJ (2002) The inflammatory reflex. Nature 420(6917):853859. https://doi.org/10.1038/nature01321

10. Koren T, Yifa R, Amer M et al (2021) Insular cortex neurons encode and retrieve specific immune responses. Cell 184(24):5902-5915.e17. https://doi.org/10.1016/j.cell.2021.10. 013
11. Gogolla N (2021) The brain remembers where and how inflammation struck. Cell 184(24):5851-5853. https://doi.org/10.1016/j. cell.2021.11.002 